\title{
RATIONALIZATION OF SAFETY FACTORS FOR BREAKWATER DESIGN IN HURRICANE-PRONE AREAS
}

\author{
Vana Tsimopoulou ${ }^{1,2}$, Wim Kanning ${ }^{2}$, Henk Jan Verhagen ${ }^{2}$ and HanVrijling ${ }^{2}$ \\ This paper presents the development of a semi-probabilistic method for armour layer design of \\ rubble mound breakwaters, which is based on the use of safety factors. The objective is to introduce \\ an approach that is both attractive to designers and sufficiently reliable when a high degree of \\ uncertainty is involved in the design process. The main focus of the analysis is the calibration and \\ appropriate use of the safety factors, which is the key element for a reliable result.
}

\section{INTRODUCTION}

One of the failure mechanisms of a rubble mound breakwater is the failure of its armour layer. In order to determine the stability of an armour layer, the design load has to be defined, which is in fact the wave that attacks the structure. Being a highly stochastic phenomenon, the wave action is not easily defined, while there is always some uncertainty inherent to its definition. In a deterministic calculation this uncertainty is being left to engineering judgment, as the possible variations of the design wave height are not taken into account in a coherent way. In order to explicitly incorporate uncertainties into the design process, and therefore increase its reliability, probabilistic design methods should be applied. A commonly used approach is a semi-probabilistic computation on level 1, which introduces the application of partial safety coefficients. Nevertheless, in the case of breakwaters, the indicated methods to derive and apply them do not clarify the uncertainties incorporated, adding an undefined degree of safety in the process, or end up with incorrect results under certain conditions (Tsimopoulou, 2010). Another approach is a fully probabilistic computation on level 2 or 3 . This type of design tackles explicitly a great deal of uncertainties, hence its results can be considered much more accurate. However it is not commonly used, due to the fact that there are not straightforward guidelines to support it, and therefore a number of critical decisions by the designers are required.

This paper presents the development of a level 1 probabilistic method for armour layer design of rubble mound breakwaters, based on the use of safety factors. The objective is to introduce an approach, which is both attractive to designers and sufficiently reliable when a high degree of uncertainty is involved in the design process. The main focus of the analysis is the calibration and appropriate use of the safety factors, which is the key element for a reliable result. For demonstration to the users the whole process is presented through an

\footnotetext{
${ }^{1}$ HKV Consultants, Botter 1129, 8232 JN Lelystad, The Netherlands

${ }^{2}$ Hydraulic Engineering, Delft University of Technology, Stevinweg 1, 2628CN Delft, The Netherlands
} 
appropriate application example. In the end a guideline for code-makers and a guideline for future designers are concluded.

An interesting case for demonstration is the jetties at the entrance of Galveston Bay, a large estuary located along the upper coast of Texas in the Gulf of Mexico (figure 1). The feature of interest on which the choice of case has been based, is the hurricane-dominated hydraulic climate of Galveston. The existence of hurricanes imposes a lot of uncertainty in the definition of the design load, which has to be taken into account. Moreover the structure is located in relatively shallow water, meaning that it is attacked by depth-limited waves, which likewise, cannot be disregarded in a design.
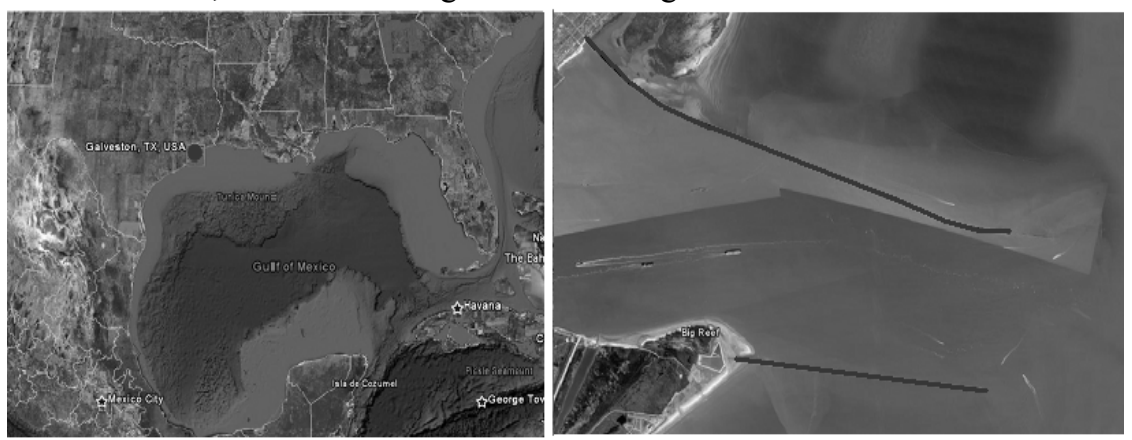

Figure 1. Location and footprint of Galveston jetties

\section{BREAKWATER DESIGN CONSIDERATIONS}

The analysis below concerns a rubble mound breakwater, which is assumed to be the optimum solution for Galveston area. The design focuses on the stability of its armour layer, which is supposed to consist of rock units. An economic lifetime for a breakwater is in the order of 50 years; hence a lifetime of 50 years is chosen. During lifetime, a probability of failure equal to $20 \%$ is assumed to be acceptable for a structure functioning as outer breakwater, like Galveston jetties. This probability corresponds to a yearly probability of failure equal to $0.4 \%$, and to a return period of design storm of 225 years. In reality the target probability of failure is chosen by means of economic optimization. This issue is out of the scope of this research, hence not elaborated. According to the above failure considerations and the available exceedance curves, the hydraulic boundary conditions can be determined. The design conditions are indicated by hurricaneinduced short waves. The stability formula of Van der Meer for plunging waves proves to be the most appropriate for this design (Verhagen, Mertens, 2007). The parameters included in this equation are described later.

$$
\mathrm{H}_{2 \%}=\mathrm{c}_{\mathrm{pl}} \cdot \mathrm{P}^{0.18}(\mathrm{~S} / \sqrt{\mathrm{N}})^{0.2}\left(\mathrm{~s}_{\mathrm{m}-1.0}\right)^{0.25} \sqrt{\cot \alpha} \Delta \mathrm{d}_{\mathrm{n} 50}
$$

The wave height $\mathrm{H}_{2 \%}$ represents the design load, and is the parameter with the highest degree of uncertainty. In the case of Galveston the water is shallow, 
meaning that only depth-limited waves can exist; hence the wave height can be approximated to a function of the water depth $h$. As water depth the total depth is considered, i.e. the depth below mean sea level $h_{d}$, plus the rise of water level due to tide $h_{t}$ and storm surge. The surge in shallow water is a function of different parameters depending on the hydraulic conditions that are examined each time. If a hurricane pass is the determining design condition, which is the case in Galveston, the surge is defined as the sum of wind set-up $h_{w}$ and pressure set-up $h_{p}$ (equation 2).

$$
\mathrm{H}_{2 \%}=\gamma_{\mathrm{b}} \mathrm{H}_{\text {surge }}=\gamma_{\mathrm{b}}\left(\mathrm{h}_{\mathrm{t}}+\mathrm{h}_{\mathrm{p}}+\mathrm{h}_{\mathrm{w}}\right)
$$

The wind and pressure set-up are functions of many other parameters, among which some hurricane parameters exist. The hurricane proves to be sufficiently described by three variables; its wind speed $u$, the angle of its trajectory $\beta$, and the distance between its landfall and the design spot $\mathrm{C}$. The wave height ends up being a function of 20 uncorrelated variables, which are presented in the table below. A detailed description of the variables can be found in the references (Tsimopoulou, 2010).

\begin{tabular}{|l|r|}
\hline \multicolumn{2}{|l|}{ Table 1. Design variables } \\
\hline$C_{p l}$ & Coefficient dependent on the wave spectrum \\
$\mathrm{P}$ & Notional permeability coefficient \\
$\mathrm{S}$ & Damage level \\
$\mathrm{N}$ & Number of waves during the design storm \\
$\mathrm{S}_{\mathrm{m}-1.0}$ & Fictitious wave steepness \\
$\mathrm{cota}_{\mathrm{a}}$ & Seaward slope of the structure \\
$\rho_{\mathrm{s}}$ & Armour unit mass density \\
$\rho_{\mathrm{w}}$ & Water mass density \\
$\mathrm{M}_{50}$ & Armour unit nominal mass \\
$\mathrm{V}_{\mathrm{b}}$ & Breaker index \\
$\mathrm{h}_{\mathrm{d}}$ & Depth below mean sea level \\
$\mathrm{h}_{\mathrm{t}}$ & Tidal elevation \\
$\mathrm{V}_{\mathrm{w}}$ & Sea water specific weight \\
$\mathrm{P}_{\mathrm{n}}$ & Atmospheric pressure \\
$\beta$ & Calibration parameter for the friction between wind and water \\
$\mathrm{C}$ & Circular hurricane speed \\
$\mathrm{C}_{1}$ & Distance between hurricane landfall and structure \\
$\mathrm{u}$ & Water depth along hurricane circular fetch \\
$\mathrm{d}$ & Gravitational acceleration \\
$\mathrm{g}$ & Angle of hurricane landfall with the normal of the coastline \\
\hline
\end{tabular}

\section{SEMI-PROBABILISTIC DESIGN FORMAT}

There are several methods for designing an armour layer, yet in all of them there is a contradiction between two equally important qualities; the reliability of the extracted results and the ease of use of the method itself. This contradiction can be the starting point for the development of a design approach, whose objective could be the optimum combination of the two qualities. In areas like Galveston, where a high degree of uncertainty is present in the design process, a sufficiently reliable result is only feasible with a probabilistic computation. In order to have an easily applicable probabilistic method, a quasi-probabilistic 
computation on level 1 is suggested, with the use of partial safety factors in the design equation.

The concept of safety factors is a certainly effective method for designs that involve a lot of uncertainty like breakwater designs, while also very practical and easy to apply. It is commonly used in building codes, such as the Eurocodes. In many cases though, the uncertainty incorporated by the factors is not well defined, leading to less reliable results. Hence special attention needs to be paid to the derivation procedure and the proper use of the factors.

The procedure to derive safety factors can be divided in four main parts, which are elaborated in the following paragraphs:

1. Choice of safety format

2. Probabilistic determination of hydraulic load

3. Calculation of safety factors

4. Sensitivity analysis

The framework of the derivation procedure has been elaborated (CIRIA, 1977) and used (PIANC, 1997) before, with a less rational perspective though regarding the design uncertainties. It is important to note that this analysis is supposed to be elaborated by code-makers rather than designers, while designers are expected to use the extracted safety factors in a proper way in order to achieve reliable designs

\section{SAFETY FORMAT}

In order to derive the safety format, the scope of the new approach needs to be defined. This is to create a reliable and handy set of safety factors, which will cover an important degree of the uncertainty inherent to the physical problem and the design. In order to have a handy tool, the number of safety factors should be reduced to the minimum possible. Its reliability can be maximized if the maximum possible degree of uncertainty is incorporated. The least number of safety factors with which the maximum degree of uncertainty is incorporated is two: one factor for the total load, $\gamma_{S}$, and one for the resistance, $\gamma_{R}$. The application of the factors should be combined with the appropriate characteristic values of the total load and resistance, which should be explicitly indicated to the designers. This issue is discussed in a latter paragraph. The stability formula takes the following form:

$$
\gamma_{R} R^{k}-\gamma_{S} S^{k} \geq 0 \Leftrightarrow R^{*}-S^{*} \geq 0
$$

where $\mathrm{R}$ and $\mathrm{S}$ are the total load and resistance respectively, while the exponents $\mathrm{k}$ and $*$ denote characteristic and design values. They are calculated as follows:

$$
\begin{gathered}
\mathrm{S}=\gamma_{\mathrm{b}} \mathrm{H}_{\text {surge }}=\gamma_{\mathrm{b}}\left(\mathrm{h}_{\mathrm{t}}+\mathrm{h}_{\mathrm{d}}+\mathrm{h}_{\mathrm{p}}+\mathrm{h}_{\mathrm{w}}\right) \\
\mathrm{R}=\mathrm{c}_{\mathrm{pl}} \mathrm{P}^{0.18}(\mathrm{~S} / \sqrt{\mathrm{N}})^{0.2}\left(\mathrm{~s}_{\mathrm{m}-1.0}\right)^{0.25} \sqrt{\cot \alpha} \Delta \mathrm{d}_{\mathrm{n} 50}
\end{gathered}
$$

All the uncertainties covered by the load safety factor are connected with the load parameters. It is important to note that there are still some uncertainties 
related to the load which are not dealt with the load safety factor, the ones inherent to the wave steepness $\mathrm{s}_{\mathrm{m}-1.0}$ and the number of waves $\mathrm{N}$. These parameters are included in the resistance term of the chosen limit state function, and therefore incorporated to the factor of resistance. Also uncertainties associated with the probabilistic model are not incorporated in the safety factors.

\section{FULLY PROBABILISTIC DETERMINATION OF DESIGN VARIABLES}

In order to derive a reliable set of safety factors, an explicit indication of the uncertainties involved in the physical problem and the design process is necessary. A rational approach to the uncertainties inherent in the design process can only be achieved through their identification and determination of their magnitude. The degree of uncertainty is reflected to the possible variations of the design parameters, which can be quantified through their probabilistic determination, i.e. by assigning a probability distribution function to every parameter. The design parameters are in fact all the variables included in equation 1. In order to assign probability distribution functions to design parameters their physical properties need to be thoroughly investigated and any relevant data in the design location to be analysed. This analysis can be found in the references (Tsimopoulou, 2010). The provided results are the core information for a fully probabilistic computation..

\section{DERIVATION OF SAFETY FACTORS}

Based on the probabilistic parameter values the safety factors can be derived. The analytical steps to be followed are described in the following paragraphs. First the safety factor for load is calculated with a procedure that is independent of the one that will be used for the safety factor of resistance. This choice is due to the fact that the uncertainty inherent in the load is much higher that this of the structural resistance. Then the resistance factor can be calibrated based on the extracted value of the load factor.

\section{Safety factor for load}

The safety factor for load is defined as the ratio of the design load $\mathrm{S}^{*}$ over the characteristic load $\mathrm{S}^{\mathrm{k}}$ :

$$
\gamma_{\mathrm{S}}=\mathrm{S}^{*} / \mathrm{S}^{\mathrm{k}}
$$

As design load the one extracted from a fully probabilistic calculation is used that is considered to be the most accurate estimation, as all uncertainties are incorporated in a satisfactory way. Using the following limit state function, and the extracted probability distribution functions as input, a Monte Carlo simulation can be elaborated in MATLAB:

$$
\mathrm{Z}=\mathrm{S}-\gamma_{\mathrm{b}}\left(\mathrm{h}_{\mathrm{t}}+\mathrm{h}_{\mathrm{d}}+\mathrm{h}_{\mathrm{p}}+\mathrm{h}_{\mathrm{w}}\right)
$$

Where $S=$ total load, and the rest of the parameters are already known. For every particular deterministic value of the total load, the outcome is a probability of 
failure, which is in fact the probability that the Z-function becomes negative. The outcome is an exceedance probability curve indicative of the design load. If in this simulation some of the variables are replaced by deterministic values, the outcome will be a different exceedance curve. This difference is indicative of the degree of uncertainty inherent to those particular variables, which is supposed to be incorporated by the safety factors. Using deterministic values for all the load variables, the total load becomes deterministic too, and the exceedance curve turns to be a straight line parallel to the $\mathrm{x}$-axis. In order to come up with a line that represents the characteristic exceedance curve of the total load, all chosen deterministic values of the variables need to be their characteristic values.

Since there is no standard rule for the choice of characteristic values, but they vary in different designs depending on the overall design approach, a choice for all the load variables is necessary, which can be reasonably substantiated. The most commonly used choices for characteristic values are either mean variable values or values with probability of non-exceedance equal to $95 \%$. For Galveston, mean values are chosen for the majority of variables, in accordance to PIANC (1987), with only exception the variables that determine the hurricane, i.e the hurricane speed $\mathrm{u}$, distance of landfall $\mathrm{C}$, and the direction $\beta$. As characteristic hurricane, the one corresponding to category 3 hurricane of the Saffir-Simpson scale is chosen, which is commonly used in the Gulf of Mexico, with a direction SE, and landfall 5 kilometres to the north of the jetties.

The design and characteristic load exceedance curves are shown in figure 3. It should be noted that the choice of characteristic values is not critical for the final design. A different set of characteristic values would result in a different set of safety factors; the same degree of uncertainty would always be incorporated though.

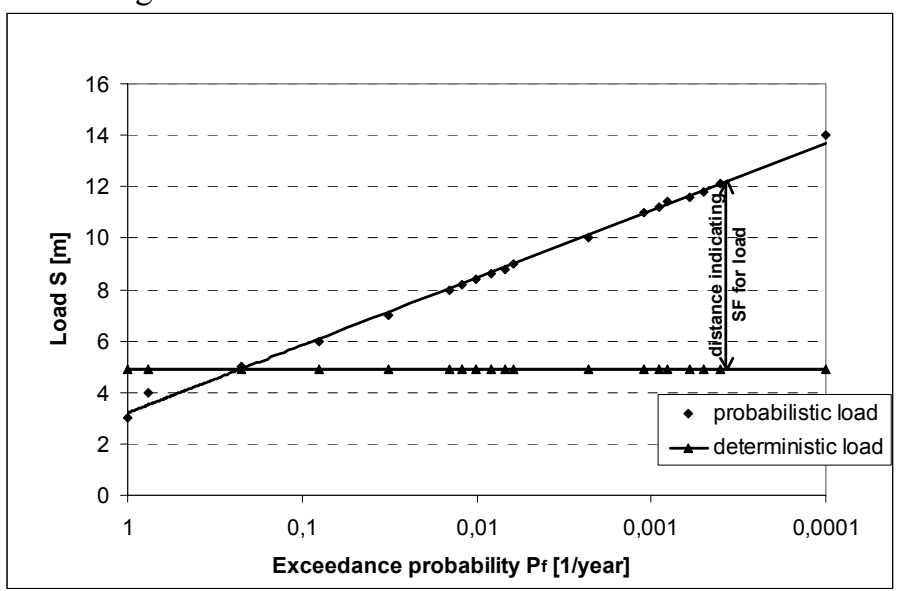

Figure 2. Design and characteristic load exceedance curves in Galveston 
Using values of the above graph, the load safety factor can be derived from equation 6. This factor accounts for the uncertainties that are neglected when the design parameters take deterministic values, and literally constitutes a measure of the divergence between the characteristic and design exceedance curves.

\section{Safety factor for resistance}

For the resistance factor a similar approach to the one adopted for derivation of the load factor is suggested. The resistance factor is meant to incorporate uncertainties inherent in the resistance part of the stability formula. An important difference between the two approaches is that in the case of resistance, the concept of exceedance curves makes no sense, as all resistance parameters are related to design and construction, and therefore their degree of randomness is a matter of choice. For this reason, the resistance factor is derived with an iterative process for certain discreet probabilities of failure, once the design hurricane and load factor are specified. This is a trial and error process applied through a Monte Carlo simulation.

The analytical steps for derivation of both load and resistance safety factors are presented below through application in Galveston.

\section{Application in Galveston}

The above-introduced procedure is applied in Galveston in order to derive a pair of safety factors for the design of Galveston jetties. The analytic calculation steps are presented below:

1. The load exceedance curve and design load are defined. For Galveston the curve corresponding to probabilistic parameter values of figure 2 is the total load exceedance curve. The target probability of failure per year is 0.0044 . According to figure 2 , this value corresponds to $\mathrm{S}^{*}=9.4 \mathrm{~m}$.

2. A design hurricane corresponding to hurricane category 3 is chosen. In fact characteristic values of the hurricane parameters need to be chosen. A hurricane of category 3 with the following characteristics is chosen as design hurricane: $\mathrm{u}=58 \mathrm{~m} / \mathrm{s}$ (category 3 ), $\mathrm{C}=5000 \mathrm{~m}, \beta=-0.75 \mathrm{rad}$.

3. The characteristic load is calculated. Substituting mean values in all load parameters and the above chosen design hurricane in equation 5 , the characteristic load shown in figure 2 is extracted: $S_{k}=4.9 m$

4. The safety factor for load is calculated with equation $7: \gamma_{S}=1.92$

5. The design load is recalculated based on the load safety factor (equation 8): $\mathrm{S}_{\text {design }}=9.41 \mathrm{~m}$

$$
\mathrm{S}_{\text {design }}=\gamma_{\mathrm{S}} \cdot \mathrm{S}_{\mathrm{k}}
$$

6. The characteristic nominal unit mass can be calculated with the Van der Meer equation, when characteristic values are substituted into all parameters (equation 9). The characteristic load has already been calculated in step 4. For the characteristic resistance, mean values of all resistance parameters are substituted into the stability formula. Those values can be found in the references. The extracted nominal mass has a very high value, $\mathrm{M}_{50}{ }^{\mathrm{k}}=33091 \mathrm{~kg}$. A rock unit of 33 tones cannot be produced and therefore 
cannot be used in the design of a rubble mound structure. For the design process this high value means that the use of artificial concrete units should be considered, as artificial units of this size can be produced and transported at the construction site. This means though that also the design stability formula has to be appropriately altered, and consequently the whole process for determination of safety factors should be repeated. Since the scope of this example is just to present the analytical steps for deriving safety factors, and not to come up with an actual design, the process will not be repeated for artificial units, but will be finalised for rock units.

$$
\mathrm{R}^{\mathrm{k}}-\gamma_{\mathrm{S}} \mathrm{S}^{\mathrm{k}}=0
$$

7. Running a Monte Carlo simulation with $\mathrm{M}_{50}=33091 \mathrm{~kg}$, mean values for all resistance parameters, and random variables for all load parameters, a probability of failure $\mathrm{P}_{\mathrm{f}}=0.004$ is calculated, which is almost equal to the target, $\mathrm{P}_{\mathrm{f} \text {,target }}=0.0044$. This result is considered satisfactory, and therefore the calculation of the load safety factor is assumed to be correct.

8. A new Monte Carlo simulation is run with the following limit state function (equation 10). This time the resistance safety factor $\gamma_{S}$ takes deterministic values, the unit mass $\mathrm{M}_{50}$ is deterministic and equal to $33091 \mathrm{~kg}$, while the remaining resistance and load parameters take probabilistic values from table 3.12. Assuming initially $\gamma_{R}=1$, a probability of failure is extracted $\mathrm{P}_{\mathrm{f}}=0.0051$, which is smaller than the target. By increasing gradually the resistance factor, the target is reached for $\gamma_{R}=1.47$

$$
\mathrm{Z}=\gamma_{\mathrm{R}} \mathrm{R}-\mathrm{S}
$$

The derived value for the resistance safety factor proves to have a quite high value, as it causes an approximately $50 \%$ increase of the design resistance, while it is only $30 \%$ lower than the load safety factor. One would normally expect a larger divergence between the two factors, as the variations of the resistance parameters, which are connected with the construction process, should be much smaller than the variations in the load, and therefore also the uncertainty inherent in the resistance should be lower than that of the load. Nevertheless this does not count in the examined case, due to the chosen safety format. The stability formula used for the design is a Van der Meer equation, which does not separate the load and resistance parameters in its two terms. This structure has been also kept in the safety format, which suggests a limit state function whose resistance term contains also three load parameters, the wave steepness, the number of waves and parameter $\mathrm{c}_{\mathrm{p} \text { l. }}$. The variation of these three parameters is the chief cause of that high value of the resistance safety factor.

The design unit mass can be easily calculated with the following equation:

$$
\mathrm{M}_{50} *=\gamma_{\mathrm{R}} \mathrm{M}_{50}{ }^{\mathrm{k}}
$$

This step is in fact part of the design process and not of the determination of safety factors. The resulting design unit mass is $\mathrm{M}_{50}{ }^{*}=48640 \mathrm{~kg}$ and converges to the result of a fully probabilistic design (Tsimopoulou, 2010). This is an 
expected result, and validates the fact that the new set of safety factors results in a design equally reliable to a fully probabilistic design.

\section{Sensitivity analysis}

The final values of the safety factors depend to an important extend on the designers' choice of characteristic values for the various variables. Through a first order reliability method simulation in MATLAB, it is concluded that the probability of failure of the structure is determined in an exceptionally high degree by two load variables, the hurricane speed $\mathrm{u}$, and the hurricane landfall parameter C. As both parameters are connected with the hurricane, their variation is very high. For this reason a sensitivity analysis is performed and different values of load safety factors are extracted for the various values of the two parameters. Some indicative results of this analysis are presented in the graphs of figure 3 .

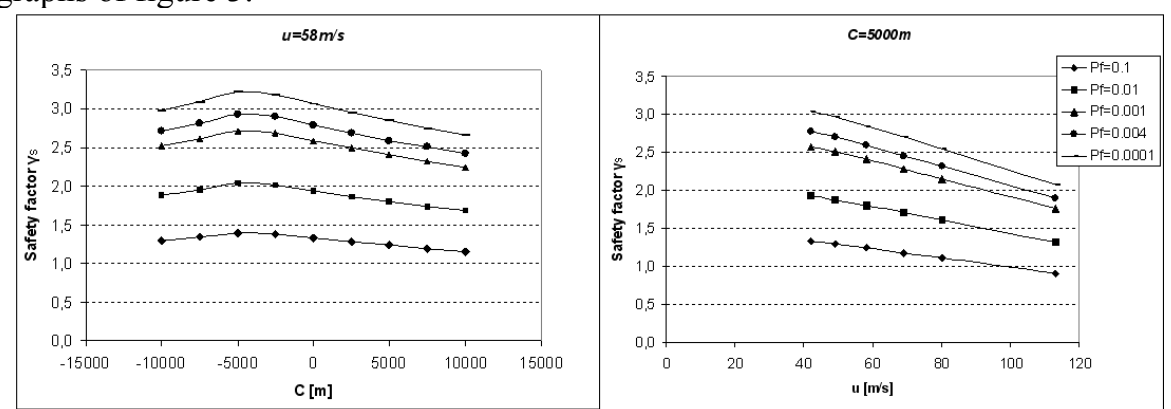

Figure 3. Load safety factor for variations of hurricane parameters in Galveston

According to the graphs the load factors increase as the target failure probability becomes lower. It is also perceptible that for higher hurricane speed the load factor decreases, meaning that when a higher design hurricane is used then a lower safety factor is needed. Both conclusions were expected. From the variations of parameter $\mathrm{C}$, the most unfavourable hurricane landfall can be concluded, which is the one requiring the highest safety factor. All curves are maximized for $\mathrm{C}=-5000$, which corresponds to a hurricane with landfall 5 kilometres to the south of Galveston jetties.

\section{GUIDELINE FOR DETERMINATION SAFETY FACTORS FOR BREAKWATER DESIGN}

The above analysis explains in detail the procedure to derive a set of safety factors. This is in fact a procedure that is not meant for designers, as it requires a Monte Carlo simulation, which is a fully probabilistic computation. This is meant for code-makers who can produce tables with appropriate safety factors for designs in different locations. The analytical steps to derive the safety factors are presented in the logical diagram of figure 4 , which can serve as a guideline for code-makers. 


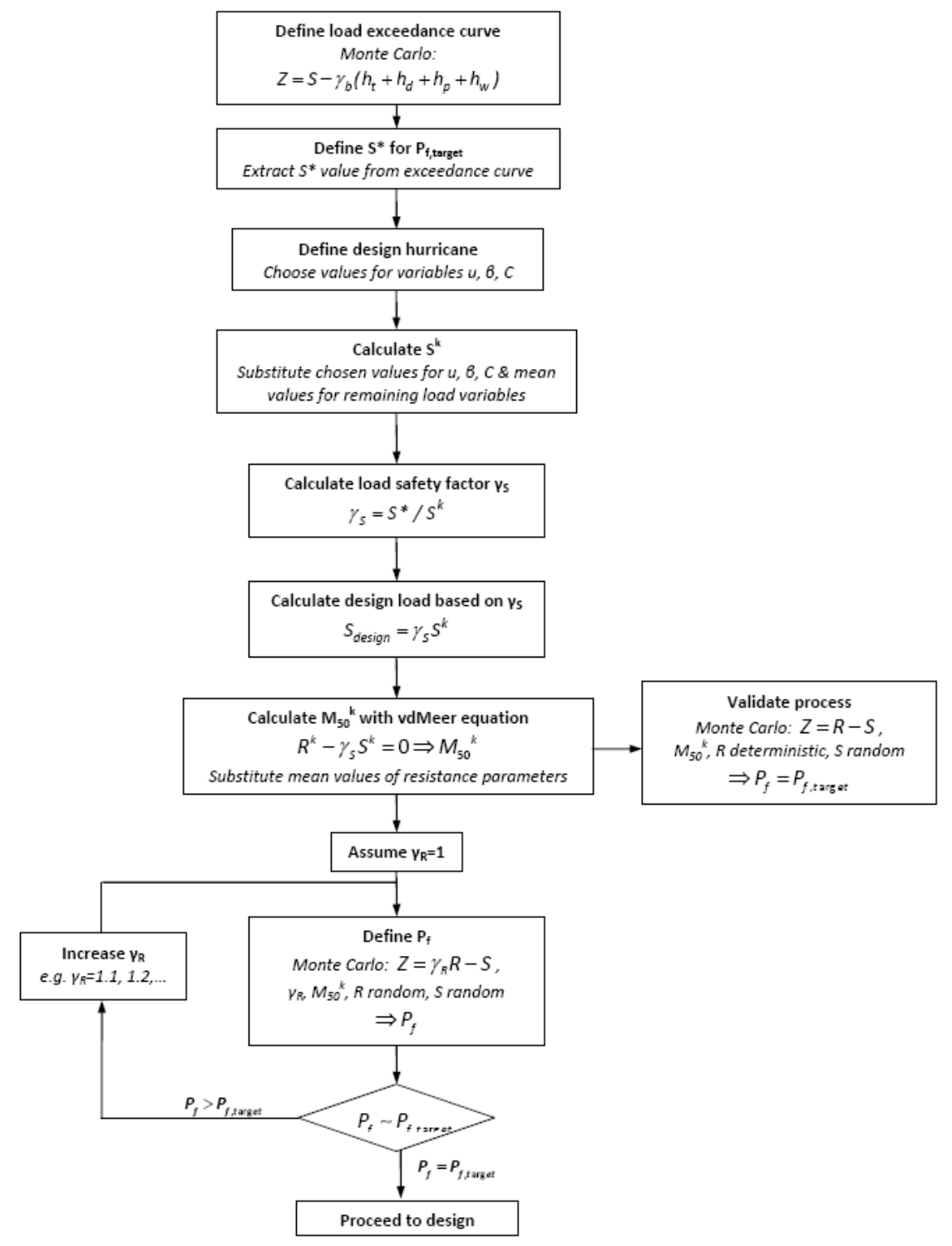

Figure 4. Guideline for code-makers

\section{EVALUATION OF ANALYSIS}

The above analysis is a rational procedure to derive safety factors for design of rubble-mound breakwaters in hurricane-prone areas. The safety factors are calibrated based on a fully probabilistic computation, for which a model describing the physical problem has been created. This model is site-specific, 
since it is developed especially for the area around Galveston. As a consequence also the results of the above determination of safety factors are site-specific, and therefore only useful in Galveston. If this analysis was generic and could be used also in other areas with the same general characteristics, then it would certainly be more valuable. There are two steps needed before the results can be used for generic breakwater design: 1) Some input parameters of the elaborated example should be reconsidered and 2) other case studies need to be conducted.

Concerning the first step, the most critical parameters that need to be reconsidered in the above-presented analysis are the ones connected with the hurricane, and especially the wind speed and parameter $\mathrm{C}$. The probabilistic description of the hurricane wind speed has been based on statistics that refer to the entire Gulf of Mexico. This is in fact a very conservative estimation, as the probability of a hurricane 3 in Galveston is much less likely than a hurricane 3 somewhere in the Gulf. For more appropriate results, the local hurricane characteristics should be used instead. This would reduce the load exceedance curve and thus lower the safety factors, and is also in line with the choice of a conservative landfall parameter $\mathrm{C}$. The same counts also for the choice of the hurricane angle $\beta$, though it affects much less the value of the load safety factor. Further it should be remarked that the safety are rather conservative due to a conservative assumption made in the early stages of this research. The hurricane speed used for deriving the local water levels in Galveston is the one-minutesustained wind speed, which is very conservative for determination of hydraulic conditions. A wind speed sustained over a few hours would be much more suitable for such calculation. This assumption has to be reconsidered before this analysis is used in practice.

The second step in order to apply the safety factors for generic breakwater design is to incorporate more case studies at different locations. As different location have different hurricane characteristics and bathymetry, the derived safety factors will have different values. The result of different case studies will be a scatter of safety factors. Subsequently, one safety factor could be chosen. If this will be the mean, highest or a quantile value depends on how bad it is perceived if a structure that is designed with the safety factor does not comply to the target probability of failure. For instance, if the mean safety factor of the different cases is chosen, this means that approximately $50 \%$ of the structures designed with this safety factor doesn't comply with the target reliability. In fact, an optimum between conservativeness, i.e. all structures have to comply, but most are too strong, and accuracy, i.e. less strong design, not all structures comply with the target reliability, has to be found.

\section{CONCLUDING REMARKS}

Based on this research a number of concluding remarks have been drawn and are summarized below.

1. A new design method is suggested which is based on the concept of safety factors. The main goal is to develop a design equally reliable to a fully 
probabilistic design. This is achieved with the definition of a set of safety factors that deals explicitly with all uncertainties inherent to the physical problem and the design itself.

2. The derivation of the new safety factors is based on a concept suggested by Ciria in 1977, which is appropriately modified to increase the reliability of the results.

3. The suggested safety format indicates the use of two safety factors, one for the total load and one the resistance, which will incorporate all uncertainties inherent to the load and resistance variables.

4. The derivation of safety factors is a generic procedure, which can be used as a guideline for code makers. However the resulting safety factors are site specific, as they are calibrated on the basis of a site-specific fully probabilistic computation. In order to derive safety factors in a new location, a reconsideration of the input parameters related is necessary.

5. The uncertainty inherent to the load is much higher than the uncertainty of the resistance factor, hence the load safety factor should be expected much higher than the resistance factor. The difference between the two factors is less important than expected though, due to the chosen safety format, which doesn't make a clear distinction between the resistance and load parameters.

\section{RECOMMENDATIONS}

1. In all stages of the performed analysis some simplified assumptions have been made, many of which have not been validated. As a consequence, the reliability of the extracted results and the follow-up conclusions can be questioned. An optimization of the total analysis is therefore necessary, which can be achieved through assessment and validation of all assumptions made.

2. A validation of the new design approach is necessary. This can be achieved by deriving safety factors in a different hurricane-prone area through application of the guideline for code makers.

- Based on the new sets of safety factors, a guideline for future designers could be developed, containing indication of safety factor values in different locations. This would ensure the correct use of the safety factors.

\section{REFERENCES}

CIRIA, 1977. CIRIA report 63: Rationalisation of safety factors in structural codes. CIRIA, London, $18-20,35-36$

Kanning W., 2005. Safety format and calculation methodology slope stability of dikes, MSc thesis, Delft University of Technology

Maaskant B., S. Van Vuren, M.J. Kallen, 2009. Typhoon-induced hydraulic boundary conditions in Vietnam, HKV.

PIANC, 1987. Risk analysis in breakwater design, report of MarCom subgroup 12c.

Verhagen H.J., M. Mertens, 2009. Rip rap stability for deep water, shallow water and steep foreshores, Proc. ICE breakwaters conference, Edinburgh.

Tsimopoulou V. 2010, Probabilistic design of breakwaters in shallow hurricane-prone areas, MSc thesis, Delft University of Technology. 